\title{
BIOMASA: CONSIDERACIONES GENERALES DE LA GASIFICACIÓN RESPECTO DE CICLOS TÉRMICOS PARA GENERACIÓN DE ELECTRICIDAD Y CALOR
}

SCOZZINA, Emilio Fabián ${ }^{1}$

\section{RESUMEN:}

La gasificación cuando es integrada junto a otras tecnologías de generación de calor y electricidad resulta de mucho interés en la diversificación de las matrices energéticas, dado que esto permite el uso de biomasa como fuente de combustible renovable, el tema de los rendimientos es discutible. El artículo se ofrece una visión de alternativas vigentes y su uso como mejores tecnologías disponibles aportando elementos para tener una visión objetiva el proceso.

Palabras clave: Biomasa, Energía eléctrica, Gasificación, Ciclos térmicos.

\section{INTRODUCCIÓN:}

La Gasificación es un proceso termoquímico en el que la biomasa, de origen leñoso o procedente de los residuos agrícolas, son transformados en un gas combustible (gas pobre). Este proceso tiene un rendimiento que depende la biomasa utilizada y del tipo tecnología de gasificador empleado en la conversión, desarrollaremos este concepto de relacionado con distintos ciclos térmicos convencionales utilizados para generación de energía eléctrica y térmica.

\section{GASFICACIÒN DE BIOMASA Y CICLOS TÉRMICOS:}

El proceso de gasificación se realiza mediante la combustión de la biomasa en una atmosfera con bajo volumen de oxígeno este proceso termoquímico genera un gas de bajo poder calorífico y como residuos se obtienen un sólido carbonoso y cenizas. Durante la reacción, se pueden describir varios fenómenos concatenados que ocurren dentro del reactor: En principio el secado de la biomasa, que se va descomponiendo térmicamente en la pirolisis, le sigue la combustión parcial (oxidación), allí se produce un craqueo de gases, donde se desprenden vapores, alquitranes y gases de los productos constitutivos de la biomasa. El proceso

1. Mgr. Ing. Emilio F. Scozzina. Director de Grupo de Investigación en Eficiencia Energética y Transferencias de Tecnologías Aplicadas FaCENyA UNNE. efscozizna@gmail.com 
de pirolisis consiste en la descomposición de la materia orgánica por acción del calor en ausencia de oxígeno y reactantes, es la etapa previa que ocurre en los procesos de gasificación y combustión. La biomasa debe ser acondicionada adecuadamente en su granulometría. Por último, ocurre la gasificación, reacciones sólido-gas o en fase gas, mediante las que el sólido remanente se convierte en gas.

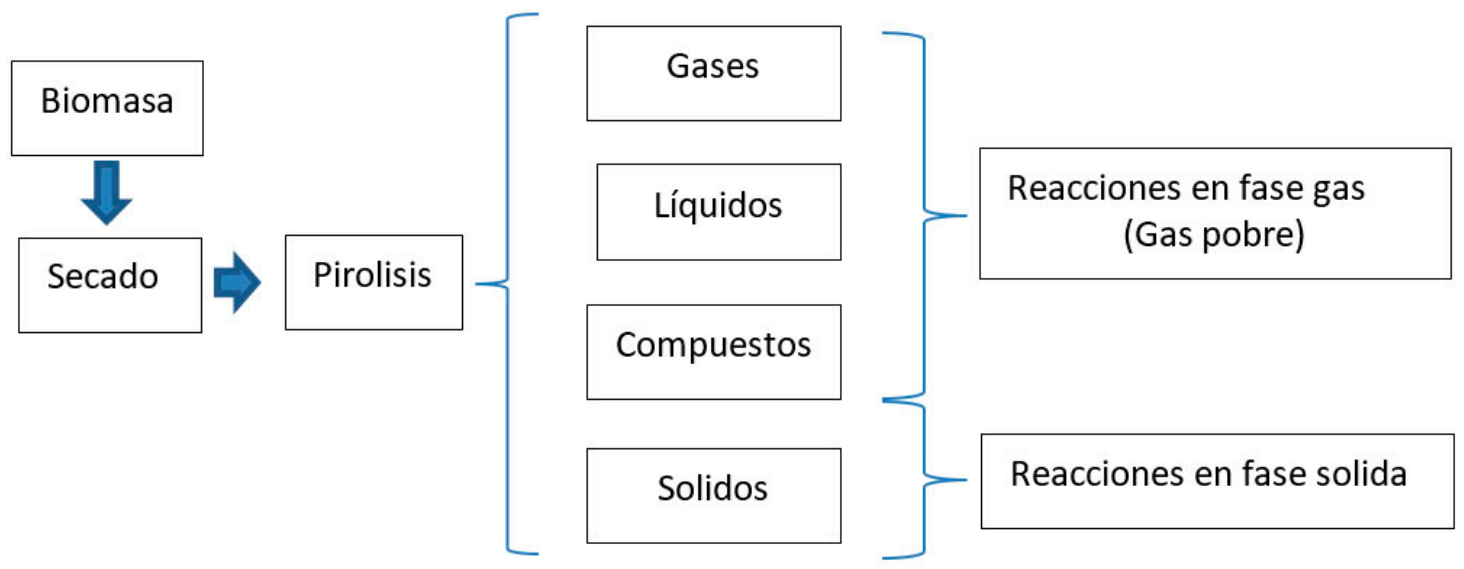

Figura $N^{\circ} 1$ Esquematización del proceso y las rutas de reacción en la gasificación.

Se tratan de reacciones fundamentalmente endotérmicas, algunas de las cuales tienen lugar en zonas determinadas del reactor. Dependiendo del tipo de gasificador, según sea de flujo ascendente o descendente (las zonas de reacción se ubicarán de diferente manera), siguiendo el diseño. El término gasificación hace referencia a una variedad de procesos en los que pueden obtenerse productos muy diversos. Una clasificación común es según el agente gasificante empleado, hay al menos cuatro tipos: gasificación con aire, con oxígeno, con vapor de agua y oxígeno (o aire) y con hidrógeno. En la Fig. No 1 se grafica el proceso de gasificación de biomasa y sus reacciones en distintas fases. $\mathrm{El}$ producto fundamental de la gasificación es el gas de bajo poder calorífico, una mezcla de $\mathrm{COx}$,
CH4, H2. Dependiendo de la tecnología del reactor y la composición de la biomasa sumado a las condiciones de operación, se generan impurezas del orden de $(10-$ $100 \mathrm{~g} / \mathrm{Nm} 3)$ tales como amoniaco, sulfuros, cloruros, junto a material particulado. Los alquitranes son compuestos orgánicos cuyo peso molecular superior al del benceno $(>76 \mathrm{~g} / \mathrm{mol})$ que generan problemas en MACI. Son mezclas complejas, propensas a la condensación a tempera ambiente y obstruyen los sistemas de tuberías, filtros, o los intercambiadores de calor que intervienen en el enfriamiento o limpieza del gas generado. En los MACI causan fallas mecánicas y reducción de la vida útil. Uno de los retos fundamentales de la gasificación es lograr eliminar de manera eficiente estos compuestos hasta los niveles permi- 
sibles. Un esquema típico de para la gasi- o MACI). Pero en una planta de producficación de biomasa está integrado por el ción de gas por estos métodos, gran parte gasificador, el sistema de limpieza de gases de la superficie de la misma es destinada a y la aplicación final (quemadores directos la limpieza y enfriamiento del gas.

\begin{tabular}{|l|l|}
\hline \multicolumn{1}{|c|}{ Aplicación } & Nivel permisible de Alquitrán \\
\hline Motor de gas & $<100 \mathrm{mg} / \mathrm{Nm3}$ \\
\hline Turbina de gas & $<50 \mathrm{mg} / \mathrm{Nm3}$ \\
\hline Turbina de combustión directa & $<5 \mathrm{mg} / \mathrm{Nm3}$ \\
\hline
\end{tabular}

Tabla $N^{\circ} 1$ Contenido de alquitranes admisibles en aplicaciones con gases de bajo poder calorifico

\begin{tabular}{|c|c|c|c|c|}
\hline $\begin{array}{l}\text { Tecnología de } \\
\text { Conversión }\end{array}$ & $\begin{array}{l}\text { Productos } \\
\text { Primarios }\end{array}$ & $\begin{array}{l}\text { Tratamiento } \\
\text { intermedio }\end{array}$ & $\begin{array}{l}\text { Conversión } \\
\text { Secundaria }\end{array}$ & $\begin{array}{l}\text { Producto } \\
\text { Final }\end{array}$ \\
\hline \multirow[t]{3}{*}{$\begin{array}{l}\text { Pirolisis } \\
\text { (Sin aire) }\end{array}$} & Carbón Vegetal & & $\begin{array}{l}\text { Caldera y Ciclo de } \\
\text { Vapor -Stirling }\end{array}$ & \multirow{8}{*}{$\begin{array}{l}\text { Electricidad } \\
\text { Calor }\end{array}$} \\
\hline & Gas Combustible & \multirow[t]{2}{*}{ Pre tratamiento } & $\mathrm{MACl}$ o Turbina de & \\
\hline & Liquido Pirolítico & & Gas & \\
\hline \multirow{3}{*}{$\begin{array}{l}\text { Gasificación } \\
\text { Aire/Vapor/ } \\
\text { Oxigeno/ } \mathrm{H}_{2}\end{array}$} & \multirow{3}{*}{$\begin{array}{l}\text { Gas combustible } \\
\text { pobre }\end{array}$} & & $\begin{array}{l}\text { Caldera y Ciclo de } \\
\text { Vapor -Stirling }\end{array}$ & \\
\hline & & \multirow[t]{2}{*}{$\begin{array}{l}\text { Limpieza del } \\
\text { Gas }\end{array}$} & $\begin{array}{l}\text { MACl o Turbina de } \\
\text { Gas }\end{array}$ & \\
\hline & & & Pila Combustible & \\
\hline \multirow[t]{2}{*}{$\begin{array}{l}\text { Combustión } \\
\text { Aire }+\mathrm{O}_{2}\end{array}$} & \multirow{2}{*}{\multicolumn{2}{|c|}{ Gases Calientes }} & $\begin{array}{l}\text { Caldera y Ciclo de } \\
\text { Vapor - }\end{array}$ & \\
\hline & & & Motor Stirling & \\
\hline
\end{tabular}

Tabla No2 Tecnologías de conversión de biomasa seca

Para poner en marcha sistemas de generación eléctrica/térmica en base a biomasa, se deben seguir las recomendaciones de las Mejores Tecnologías Disponibles (BAT, Best Available Techniques). Este grupo de procesos y tecnologías ambientalmente probadas, se pueden definir, como la manera más respetuosa de tratar con el medio ambiente al desarrollar una actividad, teniendo en cuenta que el costo que significa para las empresas que las utilizan y si se encuentran operativas dentro de unos límites razonables para su puesta marcha de manera práctica. La filosofía BAT se basa en el desarrollo sostenible, que representa la satisfacción de las necesidades de la generación presente, sin comprometer la capacidad de las generaciones futuras para satisfacer sus propias necesidades. En la tabla No2, se muestran las distintas tecnologías de conversión para biomasa seca.

\section{La Cogeneración}

Por su eficiencia toma mucha importancia, la cogeneración, es una tecnología clave que se define como es el procedi- 
miento mediante el cual se obtiene simultáneamente energía eléctrica y energía térmica útil. Si además se produce frío se llama tri generación. En la figura №2 se puede ver cómo afecta el rendimiento conjunto, respecto de un sistema convencional.
Tengamos en cuenta que una clasificación relacionada con los tamaños de las plantas generadoras, hace la siguiente distinción, la micro generación comprende potencias menores a 50KWe y la generación a baja potencia es menor a $2 \mathrm{MWe}$.

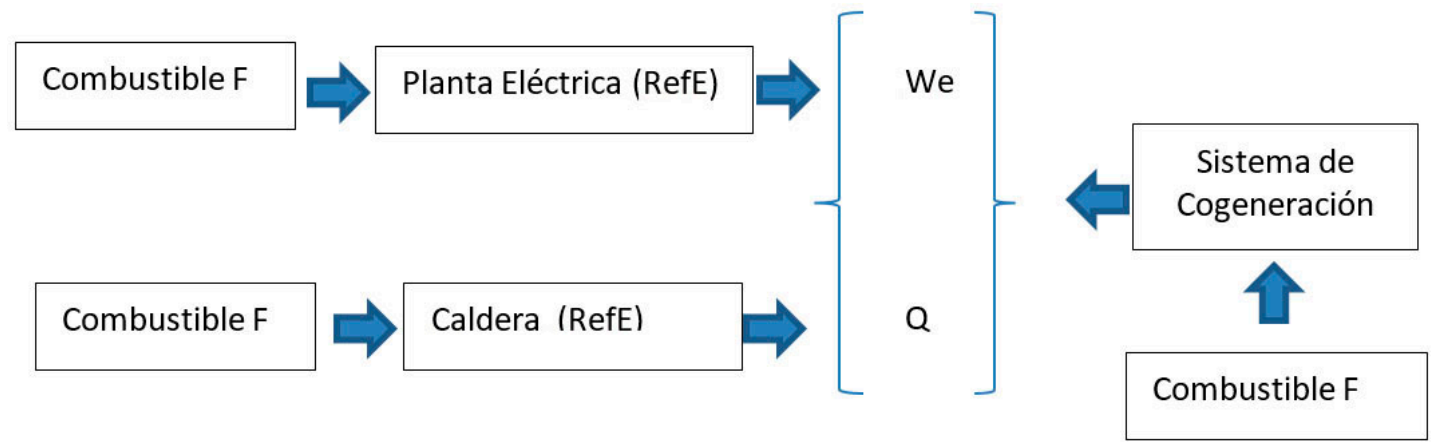

Figura No 2 Concepto de Cogeneración versus la generación individual de calor y energía eléctrica. Dónde: Energía asociada al combustible $=F$, Rendimiento eléctrico $\eta_{\mathrm{e}}=W / F$, Rendimiento eléctrico $\eta_{\mathrm{t}}=$ $Q / F$, Rendimiento energético $=\left(W_{e}+Q\right) / F$, Relación $r=W_{e} / Q$.

\section{Consideraciones Económicas}

Las consideraciones económicas para cualquier sistema cogeneración se basan en la eficiencia energética y ahorro de los combustibles frente costo de la energía eléctrica. Son factores importantes la ingeniera junto a la elección de la tecnología adecuada, la que depende de muchas variables: disponibilidad de materia prima, composición de la biomasa, dispersión, logística de recolección, políticas inversión y de desarrollo, además de considerar los elementos ambientales.

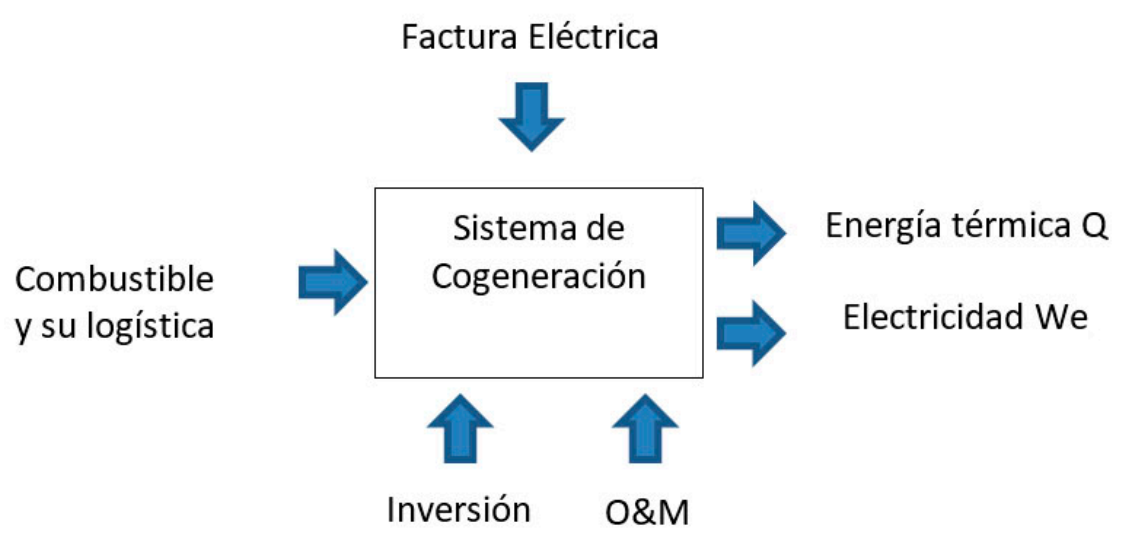

Figura $N^{\circ} 3$ Resumen de las variables económicas que se deben tener en cuenta para evaluar la implementación. 
Consideraciones del Rendimiento de un MACI (Motor a combustión interna)

Una simplificación es considerar que el rendimiento de un motor ciclo Otto, (naftero), que tiene un rendimiento que varía entre un 20 a un 30\% dependiendo de la relación de compresión y de la temperatura de funcionamiento. Esto quiere decir que un 70 a $80 \%$ de la energía suministrada por el combustible, se disipa en transformaciones energéticas indeseables, pero necesarias por el tipo de tecnología. Ej.: En los gases de escape se pierde 30\%-35\%, el calor del radiador $35 \%$ a $40 \%$, la carga del alternador requiere $5 \%$ a un $20 \%$. Si el motor se usa para accionar un generador de electricidad, el rendimiento mecánico total se debe multiplicar por el rendimiento del alternador o generador. Un generador trifásico puede tener un rendimiento de un 80 a $95 \%$. Para ejemplificar numéricamente como afecta el rendimiento de un combustible, tomemos un, $\eta \%$ $=25 \%$ para el motor naftero y un, $\eta \%=87$ $\%$ para el alternador. El rendimiento de generación medio será entonces, $\mathrm{n} \%=0,87 \mathrm{x}$ $0,25=22 \%$, como buena aproximación .

\begin{tabular}{|c|c|}
\hline \multicolumn{2}{|r|}{ Tecnología Características } \\
\hline MACI & $\begin{array}{l}\text { Tiene una aplicación para generación eléctrica con biomasa y gas pobre, van desde } \\
\text { los } 55 \mathrm{kWe}-1 \mathrm{MWe} \text {. Los rendimientos están entre el } \mathrm{n} \% 17-25 \% \text {. Las temperaturas } \\
\text { e los gases de escape pueden ir desde } 300^{\circ} \mathrm{C} \text { hasta } 500^{\circ} \mathrm{C} \text {. El radiador que enfriado } \\
\text { por agua trabaja entre } 85^{\circ} \mathrm{C}-90^{\circ} \mathrm{C} \text {, y la refrigeración de aceite lubricante es de } 85^{\circ} \text {. } \\
\text { Ventajas tiene buen comportamiento con carga, y rendimiento eléctrico aceptable, } \\
\text { (discutible). Existe disponibilidad de tecnología, tiene un coste relativo bajo en la } \\
\text { inversión. Las limitaciones son los combustibles de bajo poder calorífico y las impu- } \\
\text { rezas que arrastran, dado que afectan la vida útil del motor. La limpieza de los gases } \\
\text { de síntesis, resulta clave }\end{array}$ \\
\hline $\begin{array}{l}\text { Micro } \\
\text { Turbina }\end{array}$ & $\begin{array}{l}\text { Tiene la posibilidad de trabajar con combustibles líquidos y gaseosos, es un ciclo } \\
\text { Brayton. Para aplicaciones de biomasa manejan potencias de } 25 \mathrm{KWe} \text { a } 250 \mathrm{KWe} \text {, } \\
\text { con rendimientos de } \eta \% 15-30 \% \text {. La temperatura de los gases de escape es elevada } \\
\text { de } 400^{\circ} \mathrm{C} \text { a } 600^{\circ} \mathrm{C} \text {, es una ventaja para trabajar con ciclos combinados. Ventajas, } \\
\text { su reducido tamaño, peso y pocas partes móviles. Desventajas, se requiere personal } \\
\text { calificado para su mantenimiento y la limpieza de los combustibles sucios de bajo } \\
\text { poder calorífico sigue siendo una limitante seria. }\end{array}$ \\
\hline $\mathrm{ORC}$ & $\begin{array}{l}\text { Ciclo de Rankine, con un fluido de trabajo diferente del agua (por Ej: } \mathrm{R} 134 \mathrm{a} \text {, } \\
\text { 152a, 600). Utilizado en biomasa para los rangos de potencias que van desde los } 3,5 \\
\text {-200kW. Presenta rendimientos muy bajos } \eta \% 8 \% \text { al } 15 \% \text {. La activación de los flui- } \\
\text { dos depende de si son de bajo punto de ebullición }\left(80^{\circ} \mathrm{C} \text { y } 120^{\circ} \mathrm{C}\right) \text { o de alto punto } \\
\text { de ebullición }\left(250^{\circ} \mathrm{C} \text { a } 300^{\circ} \mathrm{C}\right) \text {. Tiene bajo mantenimiento existe una separación } \\
\text { entre el sistema de combustión y el de generación de potencia. Trabaja a bajas pre- } \\
\text { siones y presenta buen comportamiento a carga parcial. Desventajas alto costo de } \\
\text { inversión y bajo rendimiento eléctrico. Se utiliza para tri generación. }\end{array}$ \\
\hline
\end{tabular}




\begin{tabular}{|l|l|}
\hline Motor & Las aplicaciones para biomasa van desde los 3 a $150 \mathrm{kWe}$ Los rendimientos son \\
muy bajos $\eta \% 17 \%$ al $30 \%$. Ventaja hay una separación entre el sistema de combus- \\
tión el de generación de potencia, (quemador de biomasa y el Stirling). Su diseño \\
requiere una alta temperatura (foco caliente) $400^{\circ} \mathrm{C}$ a $1000^{\circ} \mathrm{C}$. Presenta una com- \\
bustión continua y bajos niveles de ruido, se comporta bien a carga parcial. Como \\
desventajas, se destaca la complejidad mecánica y el arranque lento además del \\
ensuciado del intercambiador, hay poca tecnología disponible.
\end{tabular}

Tabla No3 Comparativo de distintas tecnologías para conversión de biomasa en energía eléctrica.

\section{RESULTADOS Y DISCUSIÓN:}

Comparativos de las distintas tecnologías disponibles aplicables a la micro generación de energía eléctrica con biomasa, podemos resumir en la tabla $N^{\circ} 4$, donde se presentan sus rangos de aplicación y rendimientos asociados, según los datos proporcionados por los fabricantes.

\begin{tabular}{|c|c|c|c|}
\hline Tecnología & $\boldsymbol{\eta} \%$ & Rango de aplicación kWe & Relación Q/W \\
\hline MACl & $25-45 \%$ & $5 \mathrm{kWe}-15 \mathrm{MWe}$ & 0,28 \\
\hline Micro Turbina & $15-30 \%$ & $25-250 \mathrm{kWe}$ & 0,1 \\
\hline ORC & $8-15 \%$ & $3,5-200 \mathrm{kWe}$ & 0,35 \\
\hline Motor Stirling & $17-30 \%$ & $3-150 \mathrm{kWe}$ & \\
\hline
\end{tabular}

Tabla No 4 Tecnologias aplicables a la micro generación de con Biomasa.

De modo informativo y con números sencillos se pretende ilustrar los rendimientos asociados al proceso de gasificación y generación de energía con biomasa. Para seleccionar el proceso de gasificación adecuado hay que tener en cuenta factores como: capacidad de procesamiento, el almacenamiento, las características de la biomasa a gasificar, su granulometría, cual es el destino final de gas. Los aspectos constructi- vos son vitales, la facilidad de construcción y operación del gasificador, etc. En general todos los tipos de gasificadores pueden producir gas pobre para combustión, por ser más sencillos se prefieren los gasificadores de tiro directo en los pequeños sistemas (por debajo de una potencia térmica de 1 MW), mientras que los gasificadores de lecho fluidizado son apropiados para potencias superiores.

\begin{tabular}{|l|l|}
\hline \multicolumn{2}{|c|}{ Balance de energético de un Gasificador para un 1kg de madera } \\
\hline Combustible $\quad$ Madera & $4000 \mathrm{kcal} / \mathrm{Kg}$ \\
\hline $\begin{array}{l}\text { Perdidas: Calor de reacción termoquímica y enfriamiento del } \\
\text { gas. }\end{array}$ & $3000 \mathrm{kcal} / \mathrm{kg}$ \\
\hline Gas pobre producido & $1000 \mathrm{kcal} / \mathrm{Nm}^{3}$ \\
\hline Rendimiento de la gasificación Gas pobre limpio y enfriado. & $25 \%$ \\
\hline Rendimiento del motor y generador (Ejemplo anterior) & $22 \%$ \\
\hline Rendimiento del Gasificador, motor y generador & $22 \% \times 25 \%=5,5 \%$ \\
\hline
\end{tabular}

Tabla No 5 Resumen de rendimientos de combustible para gasificación y MACI con generador. 
Los problemas ambientales asociados a legislación vigente. No se incluyen los estas tecnologías, los que deben evaluar- asociados al MACI como ser cambios de se de manera global para poder estable- aceite y filtros y otros asociados al mantecer claramente sus ventajas, y respetar la nimiento, en la gráfica.

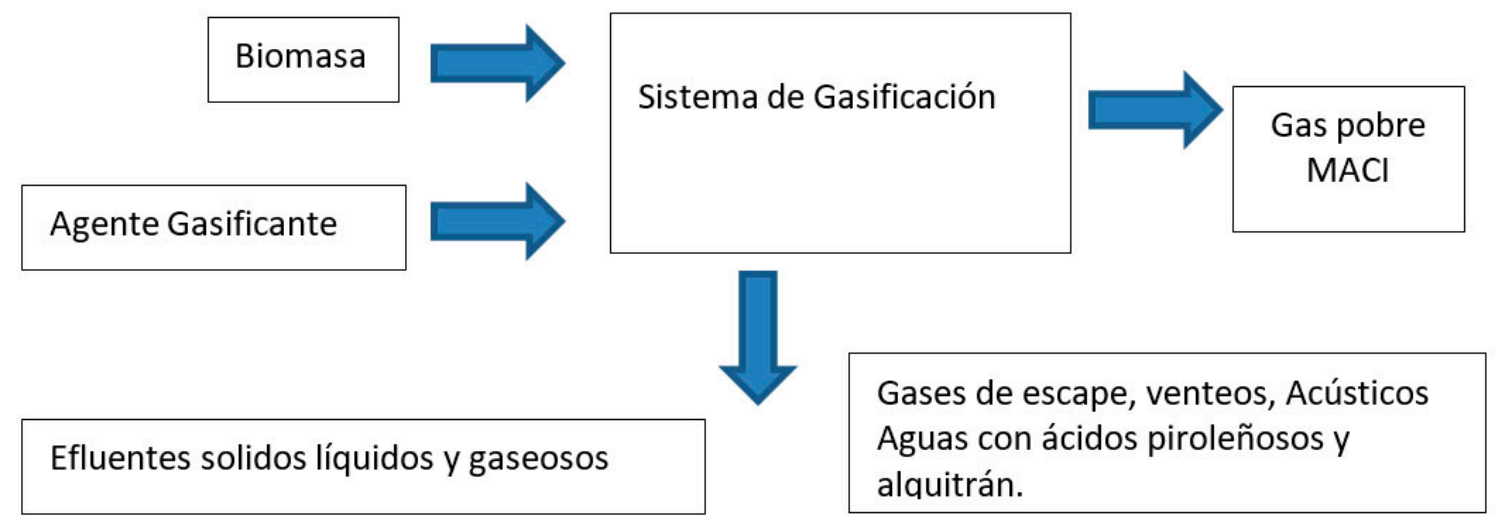

Figura $N^{\circ} 4$ Los efluentes en el proceso de gasificación. (Grafico del proceso)

\section{CONCLUSIONES:}

Se enunciaron las tecnologías básicas y fundamentales para la termo conversión de biomasa en energía eléctrica, las que se incluyen dentro de las BAT. Una de las desventajas de la gasificación de biomasa, es que el gas producido tiene un bajo poder calorífico $\left(1.000-1500 \mathrm{kCal} / \mathrm{Nm}^{3}\right)$ comparando con el gas natural $\left(9.000 \mathrm{kCal} / \mathrm{Nm}^{3}>\right)$, el butano $\left(28.000 \mathrm{kCal} / \mathrm{Nm}^{3}\right)$ o el hidrógeno $(2.500$ $\mathrm{kCal} / \mathrm{Nm}^{3}$ ). (De allí su nombre de gas pobre). Comparados con la madera (4000 kcal/ $\mathrm{kg}$ ), el rendimiento a groso modo es del $25 \%$.
La limpieza y enfriamiento del gas resulta un proceso crítico para la utilización practica en una maquina térmica. Las desventajas de la gasificación de biomasa, es que requiere de plantas industriales con altos costes de inversión y un aprovisionamiento, esta biomasa debe ser muy homogénea. Como ventaja de la gasificación, mencionamos su versatilidad, pues se obtiene energía en el momento que se requiere, a baja escala, es eficiente en la producción eléctrica o térmica, tiene un relativo bajo impacto ambiental, que debe ser considerado.

\section{LITERATURA CITADA:}

La literatura se basa en publicaciones de la Gasification and Syngas Technologies Council (GSTC), el Depósito de Documentos de la FAO sobre Gasificadores. Además se toman como referencias los siguientes autores entre otros.
REED(1998), Handbook of Biomass Downdraft Gasifier Engine System, SERIISP-271-3022 DE88001135 March 1988 UC Category.' 245 HAN KNOEF, (2005),Handbook biomass gasification 2nd Edition BTG Biomass Technology Group BV. 